CELL CYCLE

\section{The double life of IMPDH}

Mol. Cell, published online 31 May 2012,

doi:10.1016/j.molcel.2012.04.030

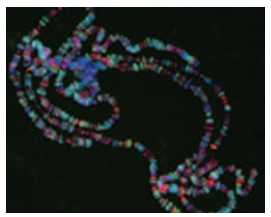

Inosine monophosphate dehydrogenase (IMPDH) catalyzes the oxidation of IMP to xanthosine monophosphate and controls the guanine nucleotide pool and therefore DNA replication and cell proliferation. Kozhevnikova et al. show that IMPDH has an additional role: it functions as a DNA-binding transcriptional repressor. In Drosophila cells after DNA replication or under oxidative stress, the authors observed that IMPDH was localized in the nucleus and was bound to chromatin. Staining of Drosophila chromosomes, chromatin immunoprecipitation (ChIP) and IMPDH knockdown experiments revealed that, at the end of S phase of the cell cycle, IMPDH binds and represses the expression of histone genes and $E 2 f$, which encodes a transcription factor important for G1-S transition and DNA replication. A genome-wide search for IMPDH binding sites by ChIP-chip technology followed by sequence alignments showed that IMPDH recognizes CT-rich loci-specifically the consensus $\mathrm{T}(\mathrm{T} / \mathrm{C})$ CTC. Previous studies have showed that IMPDH is composed of two domains: one catalytic domain and one cystathionine$\beta$-synthase (CBS) subdomain. CBS is not required for IMPDH's enzymatic activity, but it has been implicated in nucleic acid binding. By mutating either the catalytic or the CBS domain, the authors showed that the enzymatic activity of IMPDH is dispensable for DNA binding, which depends on the CBS domain. This study demonstrates that, in addition its role in nucleotide biosynthesis, IMPDH can act as a transcriptional repressor after completion of DNA replication or under oxidative stress.

AC

\section{SYNTHESIS}

\section{Cyclobutanes prove charlatans}

Angew. Chem. Int. Ed. Engl., published online 19 June 2012; doi:10.1002/anie.201203897

Angew. Chem. Int. Ed. Engl., published online 19 June 2012; doi:10.1002/anie.201203379

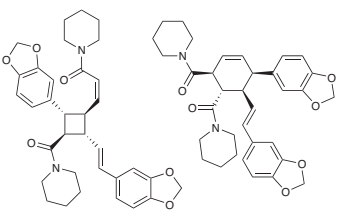

Several natural products contain cyclobutane rings thought to be biosynthesized via the $[2+2]$ cycloaddition of alkenes. This reaction has been co-opted for the chemical synthesis of several cyclobutanes but is not suitable for the preparation of unsymmetrical compounds. Two reports now describe alternate routes to the proposed structure of the unsymmetrical pipercyclobutanamide A (shown), a P450 inhibitor isolated from the flowering vines Piper nigrum and Piper chaba. In particular, Gutekunst et al. use a desymmetrized cyclobutane derivative as a precursor for arylation and a rare
METHODS

\section{How to get GAT}

ChemMedChem, published online 11 June 2012, doi:10.1002/cmdc. 201200201
MS has become a useful tool for identifying compounds that bind targets of interest in the late stages of chemical screening efforts. Sindelar and Wanner now introduce MS to earlier stages of the screening process by extending its ability to characterize single compounds in competition studies to libraries of compounds. The authors applied MS binding assays to compound libraries generated from dynamic combinatorial chemistry (DCC). Whereas 'adaptive' libraries are generated by fundamental and efficient chemical reactions in the presence of the protein target, which serves to shift the equilibrium toward the best binders, the authors use these reactions for the generation of pseudostatic libraries. The feasibility of this concept, which distinctly facilitates hit detection as library components are present in almost equal amounts, was demonstrated with the GABA transporter GAT1. The authors used DCC to generate nine small hydrazone-based libraries under conditions promoting maximal product formation and compatibility with a known GAT1 MS binding assay, which was performed in parallel. Each compound from the two most potent libraries in the binding assay was then tested individually to identify one from each library that bound GAT1 with high affinity and inhibited transporter activity. Competitive MS binding assays could prove to be a powerful readout for finding new inhibitors for unique targets. olefination of an unactivated $s p^{3}$ carbon. In contrast, Liu et al. use their recent methodology to expand a cyclopropane to a cyclobutenoate poised for conjugate addition of the final aryl substituent. Inclusion of an alkene reduction step allowed these authors to make the proposed structure for the related piperchabamide $G$ as well. Surprisingly, both papers noted that the chemical characterization of the newly synthesized molecules did not match that of the natural product. On the basis of NMR analysis and the potential for a [4+2] cycloaddition to form a cyclohexene scaffold from the same precursors, Liu et al. examined all six-membered ring products from $P$. nigrum and P. chaba. This analysis revealed pipercyclobutanamide $A$ and piperchabamide $\mathrm{G}$ to be identical to chabamide (also shown) and nigramide F, respectively, correcting the record for further biological application of these molecule.

METABOLIC DISORDERS

\section{Lipids go deaf}

Nat. Genet., published online 10 June 2012, doi:10.1038/ng.2325

MEGDEL syndrome is a newly defined metabolic disorder associated with various conditions including disturbed oxidative phosphorylation, deafness and dystonia. To understand the biochemical basis of this disorder, Wortmann et al. sequenced the exomes of two affected individuals to find mutations in a single common gene, SERAC1, whose protein product they found to be localized at the endoplasmic reticulum-mitochondria interface. The presence of a putative lipase domain was the only clue to the function of SERAC1. This, combined with the fact that MEGDEL shares phenotypes with Barth syndrome, a cardiolipin remodeling disease, led the authors to analyze phospholipids in patient fibroblasts. MEGDEL cells had elevated concentrations of the 34:1 phosphatidylglycerol (PG) and lowered concentrations of PG-36:1, resulting in an altered cardiolipin composition. Furthermore, the authors found lowered concentrations of bis(monoacylglycerol) phosphate (BMP), which led to intracellular cholesterol accumulation in patient fibroblasts. Complementation of these cells with wild-type SERAC1 could partially normalize the biochemical phenotypes. These results suggest that SERAC1 is a key player in PG remodeling in the transfer of stearic acid (18:0) to 1-palmitoyl(16:0)-2oleoyl(18:1)-PG and the as-yet-unknown BMP synthesis pathway. 
EPIGENETICS

Programming chromatin

Cell 149, 1447-1460 (2012)

Histone modifications are thought to regulate gene expression by altering chromatin structure. Hathaway et al. now report a method that allows them to establish epigenetic roles and inheritance patterns for histone marks in living cells. Their 'chromatin in vivo assay' (CiA) system builds on the principle of 'chemically induced proximity', in which a small molecule with two distinct protein-binding domains acts as a scaffold to reversibly bring proteins into proximity. In the CiA system, small molecules were used to localize chromatin-active proteins to the Oct4 promoter of mouse embryonic stem cells or somatic cells. Rapamycin-mediated localization of heterochromatin protein $1(\mathrm{HP} 1 \alpha)$ - a factor known to interact with enzymes that trimethylate Lys9 of histone $\mathrm{H} 3$ ( $\mathrm{H} 3 \mathrm{~K} 9 \mathrm{me} 3$ ) - initiated trimethylation of $\mathrm{H} 3 \mathrm{~K} 9$ repressed Oct4 gene expression. Prolonged HP1 $\alpha$ recruitment resulted in stabilized Oct4 repression as a consequence of $\mathrm{H} 3 \mathrm{~K} 9 \mathrm{me} 3$ spreading and DNA methylation at the promoter. Rapamycin washout allowed the authors to test epigenetic memory and established that the H3K9me3 mark is stably inherited across multiple cell passages. Similarly, targeting of the transcriptional activator VP12 to the Oct4 promoter by abscissic acid-mediated dimerization revealed that strongly activating complexes could override repressed chromatin states. Integrated with a kinetic modeling approach developed by the authors, the $\mathrm{CiA}$ system offers a powerful quantitative approach for inducing specific chromatin states in cells and dissecting the dynamic roles of histone modifications in gene expression regulation.

\section{SIGNALING}

\section{In Wnt's grasp}

Science, published online 31 May 2012, doi:10.1126/science.1222879

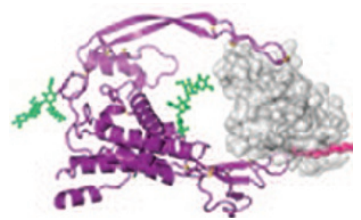

Wnts are secreted glycoproteins that mediate growth and development via multiple signaling pathways, most of which rely on interaction with a Frizzled (Fz) receptor. There are numerous Wnts and Fz receptors, so the potential for complexity in this pathway is high. There is a dearth of understanding of the specificity of Wnt-Fz interactions because little structural information is available. Janda et al. report a Wnt-Fz crystal structure, providing the first structure of any Wnt and the first ligand-bound structure of Fz. To generate sufficient amounts of the complex, the authors expressed Xenopus Wnt8 (XWnt8) together with an Fc-Fz8 cysteine-rich domain. The resulting crystals contained glycosylated Wnt and yielded X-ray data resolved to $3.25 \AA$. XWnt8 formed a unique fold, consisting of two domains, an $\mathrm{N}$-terminal $\alpha$-helical domain and a C-terminal cysteine-rich region, which interact independently with Fz8; a lipid modification on Wnt largely determined the site 1 interaction with Fz. On the basis of the structure of XWnt8, the authors engineered mini-Wnt, a water-soluble subdomain from the $\mathrm{C}$ terminus, and demonstrated that this domain, which forms site 2 , is largely responsible for discriminating among $\mathrm{Fz}$ receptors. Using surface plasmon resonance, the authors also demonstrated that mini-Wnt bound distinct $\mathrm{Fz}$ receptors with moderate but disparate affinities. Taken together, these data provide evidence for the polyspecificity of the Wnt-Fz interaction based on amino acids located at the site 1 and 2 interfaces.

\section{DRUG DISCOVERY}

\section{Polypharmacology on the fly Nature 486, 80-84 (2012)}



Although most kinase inhibitors clinically used to treat cancer were initially developed against one kinase, part of the efficacy and toxicity of many of these drugs has subsequently been attributed to blocking 'off-target' kinases. However, designing kinase inhibitors that maximize efficacy through polypharmacology while minimizing toxicity has proven challenging. To test an in vivo phenotypic screening approach, Dar et al. turned to a previously developed Drosophila model of multiple endocrine neoplasia type 2 (MEN2), a disease caused by activating mutations in RET kinase. The researchers screened a library of small molecules that inhibited RET as well as a variable spectrum of other kinases. The team next compared the phenotypic effects of the top hit, which resulted in $25 \%$ of the flies surviving to adulthood, with other library members that had a similar potency against RET but a distinct activity profile against other kinases. These data suggested that inhibiting Raf and Src increased efficacy, whereas inhibiting mTOR led to toxicity. On the basis of this information, the researchers designed a new inhibitor that rescued about $80 \%$ of the MEN2 flies and was more effective in a MEN2 mouse model than vandetanib, a kinase inhibitor approved to treat thyroid cancer resulting from RET mutations that cause MEN2. This work highlights the potential of Drosophila cancer models to facilitate the rational optimization of kinase inhibitor polypharmacology.

RNA

\section{Hitting translation first}

EMBO Rep., published online 8 June 2012, doi:10.1038/embor.2012.82

MicroRNAs (miRNAs) regulate posttranscriptional silencing by inducing deadenylation, mRNA decay and translational repression of target mRNAs. However, the kinetics and the sequence of these events are not clear. Béthune et al. now show that miRNA-mediated translational repression precedes mRNA decay and is independent of mRNA deadenylation. The authors generated HeLa cell lines expressing inducible Renilla luciferase $(\mathrm{RL})$ reporters fused to either the 3' UTR of $h m g a 2$, a target of the let7 miRNA, or to the 3' UTR of reck, a target of miR21. To gain insights into the dynamics of $\mathrm{mRNA}$ and protein silencing, they quantified the mRNA levels and luciferase activity of RL fusions carrying either wild-type or mutant (nontargeted) 3' UTRs of the miRNA targets over time. At early time points, the authors observed that the mRNA levels of wild-type and mutant reporters were comparable, whereas the amount of protein expressed from wild-type constructs was reduced compared to that from mutants, suggesting that protein translation inhibition precedes mRNA degradation. Analyses of the length of poly(A) tails of the fusion mRNAs revealed that polyadenylation was not affected during the initial steps of silencing, and knockdown of the deadenylation machinery showed that the two processes were independent. These results provide a timeline for the sequence of events by which miRNAs mediate silencing: translational inhibition precedes mRNA deadenylation and decay.

Written by Mirella Bucci, Angeliki Chalkiadaki Amy Donner, Catherine Goodman, Joanne Kotz \& Terry L. Sheppard 